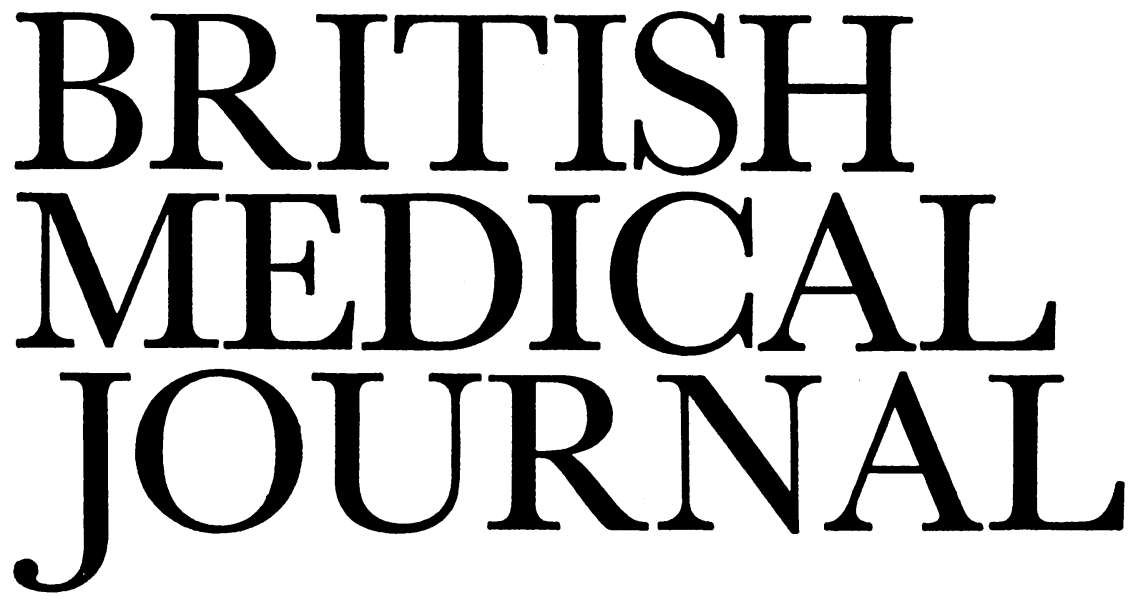

LONDON SATURDAY 8 APRIL 1972

\title{
Research in Psychiatry
}

Why has research into the nature, pathogenesis, and treatment of "functional" mental disorders made such little progress during a half-century notable for vast advances in scientific medicine generally? The paucity of resources allocated to this field of research is often blamed, yet there is no evidence that promising developments have been starved of support. In fact, the Medical Research Council and others have made great efforts to promote them.

Professor Michael Shepherd recently ${ }^{1}$ made the point that psychiatrists themselves had contributed little to research, and he puts the blame for this on two major inhibiting influences. Firstly, much psychiatric thinking, particularly in the United States, has been dominated by psychoanalytic doctrines relatively unbacked by experimental evidence and accepted on faith by other branches of medicine and the public generally. There is now a strong counterrevolution to this. Secondly, there has been the increasing recognition of the importance of sociocultural factors in psychiatric illness. Shepherd approves of this development, which he relates to patterns of medical care, but joins with D. Curran ${ }^{2}$ in attacking the expansionist tendencies of psychiatrists who digress from the investigation and treatment of patients to curing the ills of society.

Shepherd's own hopes for the future lie in two directions which are, not surprisingly, closely related to his own interests. One is the extension of epidemiological studies to establish the usually multifactorial aetiology of mental disorders from their statistical links with external circumstances, heredity, and historical events in the lives of patients. In this context the term aetiology refers only to associations. Pathogenic investigations are also needed to unravel the mechanisms of these associations. Here Shepherd pins his faith on psychopharmacological research bringing together a variety of workers in a multidisciplinary attack on relevant problems of neurobiology. This is already beginning.

But are these two lines of development enough? The statistical study of associations by skilled epidemiologists provides a rigorous extension of the classical methods of clinical research, but the nature of the associations disclosed often relates to sociological variables or the impact of stressful events such as bereavement and suggests causal mechanisms that require investigation by techniques very different from those of pharmacology. Psychopharmacological research clearly has an important role in the study of abnormal cerebral functioning but, in our existing state of ignorance, the starting points often arise from almost chance discoveries of the ameliorative properties of drugs which may be effective but not necessarily directly corrective.

Despite recent attacks on medical models of psychosis and proposals of alternative sociological models, probably there are underlying disease processes with which social factors interact. Nevertheless, it is unlikely that these processes begin with anatomical lesions or the toxic effects of pathogens, as the term "functional psychosis" implies. They may well represent inefficient or unbalanced functioning of complex adaptive psychophysiological systems in particular ecological settings. That is even more true of the common neurotic and personality disorders, which contribute greatly to our burden of sickness. They are more accurately classified as deviations than diseases, and probably represent the morbid reactions of constitutionally vulnerable individuals to varying degrees of environmental stress. ${ }^{3}$ Man is a biological organism of great complexity but he is also a product of society, playing many demanding parts, and a unique individual with a sense of identity oganized around his personal values and motives. The psychiatrist has to deal with such a peson in his entirety and in the light of his personal history and cultural background.

The complex interactions between an individual and his environment are the field of the psychologist. Just as physiology is basic to physical medicine so might psychology be considered the basic science of psychiatry. In recent decades psychology has made great advances in establishing a solid scientific foundation by rigorous experimentation. But as yet its findings seem more relevant to neurology than to psychiatry. ${ }^{4}$ The application of a strict scientific methodology has resulted in an emphasis on the experimental manipulation of isolated variables and a relative neglect of the entire dynamic system within which these variables operate. But the system as such may well be disorganized in many psychiatric disorders.

The answer to our original question therefore seems to reside in the complexity of the problems, their many-sided nature, and the high degree of hierarchical organization of human behaviour within a complex social system. Faster progress is likely to come only from a co-ordinated multidisciplinary attack to which physiologists, neurologists, psychologists, biochemists, pharmacologists, sociologists, 
anthropologists, epidemiologists, and clinical psychiatrists might contribute. Co-ordination is essential, and might best be provided by a systems analyst or other person whose specific concern is the nature of the system itself. Even if full understanding is ultimately achieved many fully developed psychiatric disorders will probably be beyond effective treatment. Research will therefore need to be directed towards early ascertainment of the vulnerable and devising prophylactic regimens which might be more akin to educational programmes than medical treatments.

1 Shepherd, M., Comprehensive Psychiatry, 1971, 12, 302. 45, 105 .

3 Jones, H. G., Bulletin of the British Psychological Society, 1971, 24, 279.

4 British Medical fournal, 1971, 4, 68.

\section{Holiday Cholera}

With the approach of the annual holiday migration from these islands to the warm south, especially to southern Europe and North Africa, we again face the risk of cholera being imported into Britain. El Tor vibrios have in the last 10 years spread from Sulawesi (formerly Celebes) across Asia and Africa and are now encroaching on the continent of Europe. It is sometimes thought that because these vibrios are different from the classical cholera vibrios they do not produce the classical clinical picture of cholera. This is not true. Untreated patients with cholera due to El Tor vibrios may die from dehydration as surely as those with the classical type of vibrio. But no one should die from cholera. It is one of the most dramatic and rewarding diseases to treat.

The diarrhoea of cholera is characteristically painless and without fever. The tissues are not invaded by organisms or toxin, and until symptoms of dehydration occur the patient feels well. All that is needed is rapid and adequate rehydration. A patient comatose and apparently moribund can within an hour or two be sitting up in bed saying that he feels fine. An adult patient in shock may take a litre of fluid intravenously in 10 minutes. Once dehydration has been corrected, and this can be assessed by return of skin turgor and a good pulse, fluid replacement must continue to balance the output of fluid in the stools. Intermittent hydration and dehydration is dangerous and may be associated with renal failure. Even adults when severely affected can become dangerously dehydrated within a few. hours; in children the danger is greater. Saline alone is not adequate as fluid replacement. Intravenous fluid must replace loss of bases and combat the acidosis. For this purpose a number of solutions are available, ${ }^{1}$ and Ringer-lactate (Hartmann's solution) is easily obtainable. Fluid replacement may continue by mouth, because provided the fluid contains about $20 \mathrm{~g}$ of glucose per litre it will be absorbed even from a gut putting out large volumes of stools. This may make considerable demands on the nurses' time, as the patient has to be kept drinking so that the intake of fluid does not fall below output. In no form of diarrhoealeast of all cholera-is there any case for withholding fluids to "dry them out" as is sometimes suggested.

A country with hygienic disposal of excreta and with a good water supply is unlikely to experience a serious epidemic of cholera, though the possibility of outbreaks must be taken seriously, ${ }^{2}$ and it is important to be prepared for adequate treatment of all cases and the surveillance of all excreters. Symptomless excreters of El Tor vibrios are important in the spread of the disease. For each case of cholera there may be several cases with mild symptoms, diagnosed perhaps as "gastroenteritis" or even a "touch of diarrrhoea." There may be many more symptomless excreters. El Tor cholera appears often to have spread by human contact and by food. It should no longer be considered as an exclusively water-borne disease. Anyone with diarrhoea returning from southern Europe or other endemic areas should be considered as possibly infected with vibrios, and specimens of faeces should be sent as quickly as possible to the nearest laboratory, which must be alerted to look for vibrios in the faeces.

Vaccination has little part to play in the control of cholera in Europe or even less developed countries. The protection from the vaccine is too short-lived. In Britain a programme of mass vaccination would be unnecessary, and in countries with poor sanitation and water supply it is, as always, a question of priorities. But anyone travelling in an endemic area may well be advised to receive immunization against cholera for his personal protection. If it is undertaken, it should be preferably with two injections spaced at least 10 days apart. Intradermal injections are not recommended. ${ }^{3}$ Vaccines, which are usually prepared from classical strains of cholera and contain a mixture of the two serotypes, Inaba and Ogawa, will give protection against $\mathrm{El}$ Tor vibrios because they are antigenically the same. But the important thing for the traveller abroad is for him to know how the disease is spread and to take proper hygienic precautions with food and water. This is more troublesome than a "shot" in the arm but more efficacious, particularly for campers and caravanners.

The insistence on a valid certificate of immunization against cholera for travellers returning to this country serves no purpose. It does not even remove the possibility that the holder is a symptomless excreter of vibrios. Some countries, including the U.S.A., no longer insist on the production of certificates of immunization against cholera from those abroad. It is time we followed suit.

1 W.H.O. Public Health Papcrs, 1970, No. 40.

3 McBean, A. M., e: al., Lancet, 1972, 1, 527.

\section{Phototherapy in Neonatal Jaundice}

Phototherapy for the prevention and treatment of hyperbilirubinaemia in the newborn is being increasingly used, but its place in management is still controversial. Its mode of action, limitations, and possible hazards have been recently reviewed ${ }^{1-4}$ and were also the main topic of a symposium on bilirubin metabolism in the newborn. ${ }^{5}$

Phototherapy probably acts by oxidizing bilirubin, and it may also cause hepatic excretion of unconjugated bilirubin. ${ }^{6}$ It can prevent hyperbilirubinaemia in premature infants, ${ }^{78}$ but anxiety about the effects of the photodegradation products of biliribin and ignorance of its long-term sequelae have made paediatricians hesitate to use this form of 